\title{
BMJ Open Cost-effectiveness of a quality improvement programme to reduce central line-associated bloodstream infections in intensive care units in the USA
}

To cite: Herzer KR,

Niessen L, Constenla D0, et al. Cost-effectiveness of a quality improvement programme to reduce central line-associated bloodstream infections in intensive care units in the USA. BMJ Open 2014:4:e006065.

doi:10.1136/bmjopen-2014006065

- Prepublication history and additional material is available. To view please visit the journal (http://dx.doi.org/ 10.1136/bmjopen-2014006065).

Received 9 July 2014 Revised 15 August 2014 Accepted 3 September 2014

CrossMark

For numbered affiliations see end of article.

Correspondence to Kurt Herzer; kherzer@jhmi.edu

\section{ABSTRACT}

Objective: To assess the cost-effectiveness of a multifaceted quality improvement programme focused on reducing central line-associated bloodstream infections in intensive care units.

Design: Cost-effectiveness analysis using a decision tree model to compare programme to non-programme intensive care units.

Setting: USA.

Population: Adult patients in the intensive care unit. Costs: Economic costs of the programme and of central line-associated bloodstream infections were estimated from the perspective of the hospital and presented in 2013 US dollars.

Main outcome measures: Central line-associated bloodstream infections prevented, deaths averted due to central line-associated bloodstream infections prevented, and incremental cost-effectiveness ratios. Probabilistic sensitivity analysis was performed.

Results: Compared with current practice, the programme is strongly dominant and reduces bloodstream infections and deaths at no additional cost. The probabilistic sensitivity analysis showed that there was an almost $80 \%$ probability that the programme reduces bloodstream infections and the infections' economic costs to hospitals. The opportunity cost of a bloodstream infection to a hospital was the most important model parameter in these analyses.

Conclusions: This multifaceted quality improvement programme, as it is currently implemented by hospitals on an increasingly large scale in the USA, likely reduces the economic costs of central line-associated bloodstream infections for US hospitals. Awareness among hospitals about the programme's benefits should enhance implementation. The programme's implementation has the potential to substantially reduce morbidity, mortality and economic costs associated with central line-associated bloodstream infections.

\section{INTRODUCTION}

Central line-associated bloodstream infections (CLABSI) are common, expensive to

\section{Strengths and limitations of this study}

- This study was conducted according to best practices in cost-effectiveness analysis and demonstrates that a multifaceted quality improvement programme can reduce the economic costs of central line-associated bloodstream infections for hospitals.

- We used nationally representative data sources to increase generalisability and performed a probabilistic sensitivity analysis to quantify the uncertainty in our cost-effectiveness estimates.

- Owing to data limitations we were unable to assess the impact of patient heterogeneity, such as demographics and clinical characteristics, on baseline risk, treatment effect or resource utilisation. We did not evaluate costs outside the acute hospital setting, such as rehabilitation costs or productivity losses for delays in returning to work.

payers and patients, and potentially fatal. ${ }^{1}{ }^{2}$ Each year, nearly 80000 Americans develop CLABSIs in intensive care units (ICU), and more than 25000 of these patients die. ${ }^{3}$ A single infection can cost payers as much as $\$ 56000$, culminating in over $\$ 2$ billion in related costs per year in the USA. ${ }^{4}$ CLABSIs in ICU patients have an estimated attributable mortality rate of $14-40 \%$, with a prolonged length of stay of 7.5-25 days. ${ }^{5} 6$

The Keystone ICU project, first launched in Michigan in 2004 and since scaled across the USA, Spain, Peru, Pakistan and the UK, has captured the interest and attention of patients, payers and policymakers for its substantial, sustained and scalable reductions in preventable nosocomial infections. Over 1200 US hospitals are currently participating in this multifaceted quality improvement programme through On the CUSP: Stop BSI, 
a national collaborative, and many others are likely using checklists and infection prevention programmes in their ICUs as standard practice. The programme has been evaluated through prospective cohort studies, ${ }^{7-10}$ retrospective observational studies using claims data, ${ }^{11}$ and both cluster non-randomised ${ }^{12}$ and randomised controlled trials. ${ }^{13}$ When viewed collectively, this evidence suggests that the programme is associated with substantial reductions in CLABSIs and mortality in ICU patients.

In spite of commendable investment in this programme to manage the undesirable consequences of CLABSIs, an important question remains unanswered: compared with current practice, is this programme costeffective for US hospitals? Reporting of economic data in quality improvement studies is uncommon, and there are few formal cost-effectiveness analyses of quality improvement programmes. ${ }^{14-16}$ Similarly, because the estimated gross costs of CLABSIs to the healthcare system are very high, the conclusion that expanding infection control efforts will be cost saving (relative to the costs incurred by expanded efforts) is accepted without rigorous analysis. ${ }^{17}$ This paper examines the cost changes and cost-effectiveness of the Keystone ICU project from the perspective of the hospital using nationally representative data sources from the USA.

\section{METHODS}

\section{Overview of the analysis}

We developed a decision tree model to address the choice faced at an individual hospital about implementing the programme (figure 1). The use of a decision tree approach is justified by the short-term progression of CLABSIs. The model assumes that patients do not experience other adverse effects of catheterisation, such as catheter colonisation leading to local infection, hypersensitivity reactions or mechanical complications such as pneumothorax. The Keystone ICU project instead focused on infectious complications, because they are more common, more costly and often fatal. ${ }^{18}$ Consistent with other economic evaluations of CLABSIs in the ICU setting, we assumed that the consequences of infection are independent of age, patient disease severity and the causative organism. ${ }^{18} 19$ These assumptions are congruent with the programme itself, which does not discriminate between subgroups of patients based on these factors.

The comparator was current practice as the most realistic alternative faced by organisations seeking to implement the programme. Current practice encompasses on-going or existing activities that might influence the risk of infection among patients, such as the use of antiinfective central venous catheters.

The target population consisted of adult (18 years or older) ICU patients in accordance with studies of the Keystone ICU project and its subsequent replications. ${ }^{13}$ Since costs and benefits only accrue while the programme is implemented, the time frame and analytic horizon are fundamentally the same. We used a time horizon of 5 years. This analysis was performed from the hospital's perspective. Our study aims to address the following question: is implementation of this quality improvement programme to decrease CLABSIs in the ICU a cost-effective approach when compared with current practice in US hospitals?

\section{Quality improvement programme}

Details of the programme and its conceptual approach have been described elsewhere. ${ }^{20-22}$ In brief, this multifaceted programme employed clinician communication tools, teamwork, and safety culture assessment and improvement tools (known as the Comprehensive Unit-based Safety Program (CUSP)), and a five-item, evidence-based checklist for correctly inserting central venous catheters. The five components of the checklist included using basic hand hygiene, exercising full barrier precautions, cleaning the skin with chlorhexidine, avoiding the femoral site when possible, and removing any unnecessary catheters. A model for
Figure 1 Decision tree model. Decision tree model depicting programme versus no programme and its effects on outcomes in intensive care unit (ICU) patients. 'Bloodstream infections' refers to central line-associated bloodstream infections.

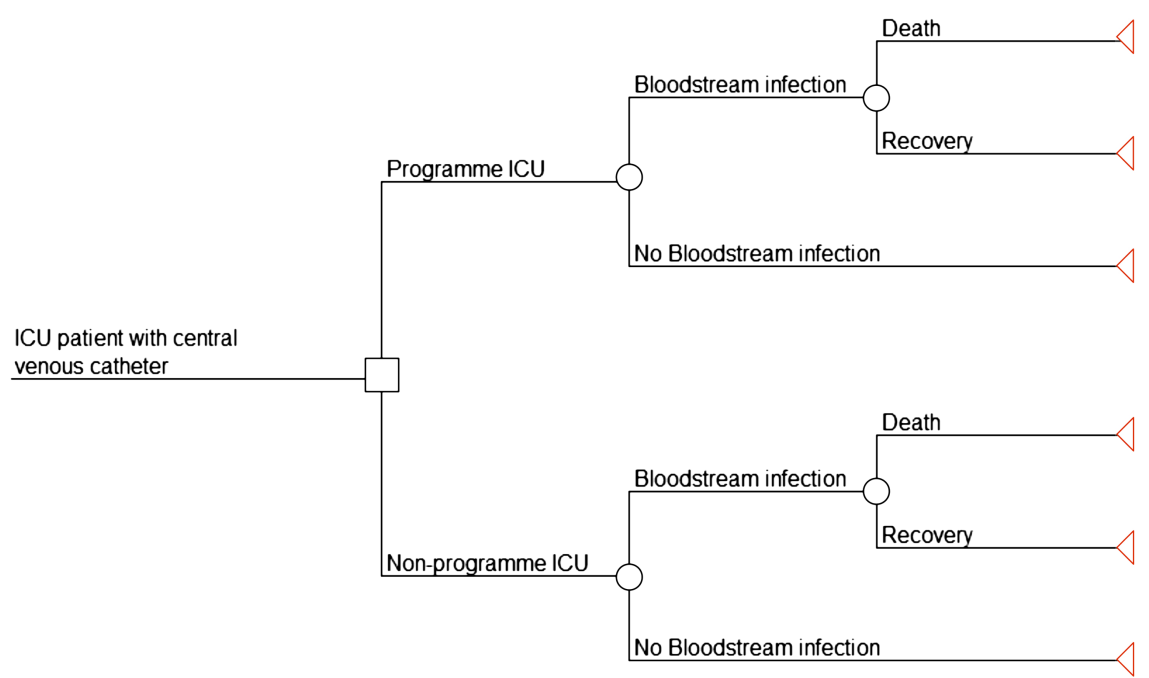


translating evidence into practice identified and mitigated local barriers to implementation of the checklist. ${ }^{22}$ Quarterly infection rates measured at the ICU level were used to monitor progress towards the goal of reducing CLABSIs.

\section{Model inputs}

Table 1 shows the key parameters used in the decision tree model, such as probabilities and cost inputs as well as the effectiveness of the programme, which are described in detail in the following sections.

\section{Risk of CLABSI and death}

Estimates of the risk of CLABSI given exposure to a central venous catheter varied from $3.0 \%$ to as much as $16.0 \% .^{23}{ }^{24}$ We used a probability estimate of $5.2 \%$ for a standard catheter, derived from a meta analysis of 13 randomised controlled trials from a previous economic evaluation. ${ }^{18}{ }^{25}$ Estimates of the attributable mortality of CLABSI ranged from $14 \%$ to $40 \% .^{5} 62627$ We used a point estimate of $15 \%$.

\section{Costs}

Estimates of payer costs attributable to CLABSI varied widely, from as little as $\$ 6000$ to over $\$ 56000{ }^{4} 61828$ The reasons for this variation can be attributed to the small sample sizes of studies, challenges allocating inpatient costs, perspectives used, the types of cost categories included, and the methodology used for estimation. ${ }^{29}$ Existing studies have largely focused on financial costs (or hospital charges or payer costs) rather than opportunity costs and so they may incorrectly estimate the economic cost of CLABSIs to hospitals. We considered the economic cost of CLABSIs in terms of the increased length of stay and the variable costs associated with that occurrence. ${ }^{30}$ Given that a significant amount

\begin{tabular}{|c|c|c|}
\hline Parameter & Distribution & Source \\
\hline Probability of CLABSI* & $\beta: 0.052$ (SD 0.0074) & 1825 \\
\hline $\begin{array}{l}\text { Death attributable to } \\
\text { CLABSI }\end{array}$ & $\beta: 0.15$ (SD 0.056) & 6 \\
\hline $\begin{array}{l}\text { Incidence rate ratio of } \\
\text { programme versus } \\
\text { non-programme ICUs }\end{array}$ & $\begin{array}{l}\text { Lognormal based on } \\
\text { normal mean } 0.19 \\
\text { (SD 0.13) }\end{array}$ & 13 \\
\hline \multicolumn{3}{|l|}{ Total cost\$ } \\
\hline CLABSI (per patient) $†$ & $\begin{array}{l}\text { Lognormal based on } \\
\text { normal mean } \\
\$ 18793 \text { (SD 5533) }\end{array}$ & 33 \\
\hline $\begin{array}{l}\text { Programme } \\
\text { (per patient)† }\end{array}$ & $\begin{array}{l}\text { Lognormal based on } \\
\text { normal mean } \$ 540 \\
\text { (SD 120) }\end{array}$ & 29 \\
\hline \multicolumn{3}{|c|}{$\begin{array}{l}{ }^{*} \text { Conditional probability of a CLABSI given exposure to a central } \\
\text { venous catheter, assumes standard (non-antimicrobial) catheter. } \\
\text { †Discounted costs presented. } \\
\text { CLABSI, central line-associated bloodstream infection; ICU, } \\
\text { intensive care unit. }\end{array}$} \\
\hline
\end{tabular}

of hospital costs are fixed in the short run, the economic viability of quality improvement programmes that reduce CLABSIs rests on two things: deploying the bed-days freed by shorter lengths of stay for new admissions and reducing utilisation of medications and supplies. The value of the new admissions (the potential incremental net revenue opportunity per prevented infection) represents the economic cost of infection and accordingly, the potential economic cost avoidance resulting from infection prevention. ${ }^{31}$ Using this approach, we estimated that the discounted cost of a CLABSI was \$18 793 (see online appendix tables A and $\mathrm{B}$ for details of this calculation).

Start-up costs and recurring costs associated with the quality improvement programme were assigned using an activity-based microcosting of the programme performed in six hospitals in Michigan. ${ }^{29}$ We assumed that start-up costs occurred in the first year of implementation and did not discount them. Capital items, such as bloodstream infection line carts, were annualised assuming a 5 -year useful life and 3\% discount rate. We included the opportunity costs of key personnel whose time was committed to the programme even though a hospital may not incur any financial costs related to personnel who are already on staff. We estimated this cost by multiplying each staff person's percentage effort committed to the programme by an estimate of that position's annual compensation. We used the Society of Critical Care Medicine's annual compensation estimates from 2009 for critical care physicians, nurses, pharmacists and respiratory therapists. ${ }^{32}$ The salary for infection control preventionists was based on the Bureau of Labour Statistics's 2011 Occupational Employment Statistics and we added $30 \%$ benefits. Table 2 presents a detailed itemisation of the start-up costs and recurring costs of the programme. Start-up costs were approximately $\$ 80000$ and recurring costs were approximately $\$ 192000$ per hospital per year. Personnel costs were the largest contributor, comprising $95 \%$ of start-up costs and $89 \%$ of recurring costs.

We estimated a per patient cost of the programme by deriving an average number of ICU patients per hospital who had central venous catheters (the patients most likely to benefit from the programme's prevention efforts). To calculate this, we first derived a national annual cohort of ICU patients exposed to central venous catheters by multiplying the total annual ICU admissions in the USA by an estimate of the proportion of patients admitted to an ICU that receive central venous catheters. ${ }^{33}$ We then divided this group of ICU admissions with central venous catheters by the number of hospitals that reported having adult ICUs in the American Hospital Association Annual Survey of Hospitals, ${ }^{34}$ yielding an average number of 'exposed' patients per hospital. Finally, we divided the total costs of the programme per hospital by the number of patients per hospital to yield an average cost for the programme per patient of $\$ 540$ (SD, 120). Online appendix table $\mathrm{C}$ contains additional 
Table 2 Itemisation of programme costs (per hospital)

\begin{tabular}{|c|c|c|}
\hline Cost category & Start-up costs & Recurring costs \\
\hline \multicolumn{3}{|l|}{ Personnel\$ } \\
\hline Critical care physicians ( 2 on average per hospital) & $\$ 26004$ & $\$ 71953$ \\
\hline Nurses (8 on average per hospital) & $\$ 44406$ & $\$ 75306$ \\
\hline Respiratory therapists & $\$ 4605$ & $\$ 7923$ \\
\hline Infection control preventionists & $\$ 1981$ & $\$ 7855$ \\
\hline Pharmacists & $\$ 2725$ & $\$ 7962$ \\
\hline \multicolumn{3}{|l|}{ Education and Training\$ } \\
\hline Education and training expenses & $\$ 3579$ & \\
\hline \multicolumn{3}{|l|}{ Capital items\$ } \\
\hline CLABSI line cart/central line insertion cart (annual equivalent cost) & $\$ 426$ & $\$ 426$ \\
\hline \multicolumn{3}{|l|}{ Materials\$ } \\
\hline Chlorhexidine & & $\$ 2378$ \\
\hline Oral care kits & & $\$ 6933$ \\
\hline Sterile central line dressing kits & & $\$ 11555$ \\
\hline Total\$ & $\$ 83725$ & $\$ 192292$ \\
\hline
\end{tabular}

details of this calculation, including the uncertainty ranges incorporated into the estimate.

All costs were adjusted to 2013 US dollars using the consumer price index for medical care services. Recurring costs were discounted by $3 \%$ annually. In separate sensitivity analyses, we examined the effect of not discounting costs and of discounting costs by $5 \%$.

\section{Effectiveness}

We based our estimate of the programme's effectiveness on a cluster randomised controlled trial that found an $81 \%$ reduction in CLABSI rates comparing programme ICUs with control ICUs (incidence rate ratio, 0.19; 95\% CI 0.06 to 0.57$).{ }^{13}$ We used the CI of this point estimate, a measure of uncertainty in the programme's effectiveness, to derive a SD of the estimate for probabilistic sensitivity analysis. This effectiveness parameter was measured at the ICU level but in our model we assumed that it applied to individual patients. This ecological assumption was necessary because there are no patient level effectiveness estimates available from the Keystone ICU project. The SD of the estimate captures the heterogenic reality that the intervention benefit is not uniform among ICU patients.

To calculate the base case probability of a CLABSI in the programme arm of the decision tree, we multiplied the probability of CLABSI by the incidence rate ratio (0.19) from the trial. We assumed that by the end of the first year of implementation, programme ICUs achieve this reduction. The original ICUs that implemented the programme maintained the reduction for the subsequent 10 years (Sam Watson, written communication, 18 August 2013). We assumed this to be true for the 5-year period used in this evaluation. ${ }^{35}$

The number of annual ICU admissions requiring catheters, the probability of infection and the attributable mortality parameters were assumed not to change during the 5-year period.

\section{Outcome measures}

The two outcomes of interest were CLABSIs prevented and deaths averted (lives saved). We calculated the number of CLABSIs prevented as the difference in the number of CLABSIs between programme and nonprogramme ICUs. Deaths averted were calculated similarly. We did not discount CLABSIs or deaths in the base case analysis to avoid making the ethically challenging assumption that infections or deaths prevented in the future are worth less than they are in the present. We explored the effect of discounting CLABSIs and deaths by $3 \%$ in a sensitivity analysis.

We calculated incremental cost-effectiveness ratios as the additional cost divided by the additional health benefit (CLABSIs prevented or deaths averted) comparing the programme to current practice.

\section{Probabilistic sensitivity analyses}

We conducted a probabilistic sensitivity analysis to account for uncertainty in the model's input parameters. We used Monte Carlo simulation to perform 10000 iterations of the model, simultaneously sampling each parameter from an underlying distribution that reflects the degree of uncertainty in the parameter estimate. Uncertainty in parameter estimates was obtained from the CIs or SDs published with the point estimates. Table 1 presents the modelled distributions, point estimates and SDs for the key model parameters. All analyses were performed using Microsoft Excel (Microsoft Corporation; Redmond, Washington, USA) with @Risk (Palisade Corporation; Ithaca, New York, USA). 
Table 3 Comparison of costs and outcomes between programme and non-programme ICUs

\begin{tabular}{|c|c|c|c|}
\hline & Mean & Median & 2.5th-97.5th centile \\
\hline \multicolumn{4}{|l|}{ Non-programme ICU } \\
\hline CLABSIs & 52 & 52 & $39-66$ \\
\hline Deaths & 8 & 8 & $2-14$ \\
\hline Costs* & $\$ 987000$ & $\$ 937000$ & $\$ 488000-\$ 1760000$ \\
\hline \multicolumn{4}{|l|}{ Programme ICU } \\
\hline CLABSIs & 10 & 9 & $3-29$ \\
\hline Deaths & 2 & 1 & $0-5$ \\
\hline Costs $\$ *$ & $\$ 738000$ & $\$ 710000$ & $\$ 453000-\$ 1190000$ \\
\hline \multicolumn{4}{|l|}{ Benefit of programme } \\
\hline CLABSIs prevented & 42 & 42 & $23-58$ \\
\hline Deaths averted & 6 & 6 & $2-12$ \\
\hline Net costs\$ & $-\$ 249000$ & $-\$ 221000$ & $-\$ 976000$ to $\$ 300000$ \\
\hline \multicolumn{4}{|c|}{ Incremental cost-effectiveness ratio (probability) } \\
\hline Cost per CLABSI prevented & \multicolumn{3}{|c|}{ Strongly dominant $(0.80) \ddagger$} \\
\hline Cost per death prevented & \multicolumn{3}{|c|}{ Strongly dominant $(0.80) \ddagger$} \\
\hline \multicolumn{4}{|c|}{$\begin{array}{l}\text { Mean, median, } 2.5 \% \text { and } 97.5 \% \text { centile estimates for outputs from probabilistic sensitivity analysis of } 10000 \text { model runs representing } \\
\text { uncertainty in epidemiological and economic parameters are reported. } \\
\text { All mean, median, and centile values are expressed per } 1000 \text { patients to make the scale easier to interpret. Values have been rounded to } \\
\text { three significant digits at most. } \\
{ }^{*} \text { Costs are not presented separately for each outcome (CLABSI and death) because no additional cost was assumed to occur for death; } \\
\text { discounted at } 3 \% \text {. } \\
\text { †Benefit of programme determined by subtracting programme ICU estimates from non-programme ICU estimates within the model. } \\
\text { †Probability that the programme is more effective and less costly than current practice. } \\
\text { CLABSI, central line-associated bloodstream infection; ICU, intensive care unit. }\end{array}$} \\
\hline
\end{tabular}

\section{RESULTS}

Table 3 compares programme and non-programme ICU costs and outcomes for CLABSIs prevented and deaths averted. The quality improvement programme prevents 42 CLABSIs per 1000 patients and averts 6 deaths per 1000 patients at no additional cost in the base case analysis, representing a dominant strategy when compared with current practice.

Figure 2 and online supplementary figure S1 show the cost-effectiveness planes comparing the joint density of incremental costs and incremental effects for 10000 model iterations. Incremental refers to the difference in costs or effects between programme and nonprogramme ICUs. The X-axis represents the incremental level of effectiveness of an outcome and the Y-axis represents the additional total cost of achieving this outcome. Each data point in the scatterplot represents an estimated incremental cost-effectiveness ratio for the outcome; as such, the scatterplot illustrates the distribution of incremental cost-effectiveness ratios over a sample population. Points falling to the right of the Y-axis demonstrate that the programme is effective for preventing CLABSIs and averting deaths. Points falling above the $\mathrm{X}$-axis represent the additional costs of the programme and points falling below the $\mathrm{X}$-axis represent the economic cost savings from the programme. In figure 2, because $80 \%$ of the points fall below the $\mathrm{X}$-axis, there is an $80 \%$ probability that the programme reduces bloodstream infections and the infections' economic costs to hospitals compared with current practice. Figure 2 also demonstrates the presence of few extreme values (outlier incremental cost-effectiveness ratios) in the model, indicating little uncertainty in the estimates of the quality improvement programme's costeffectiveness compared with current practice. Outliers add variability and uncertainty to the overall costeffectiveness results and the existence of few such

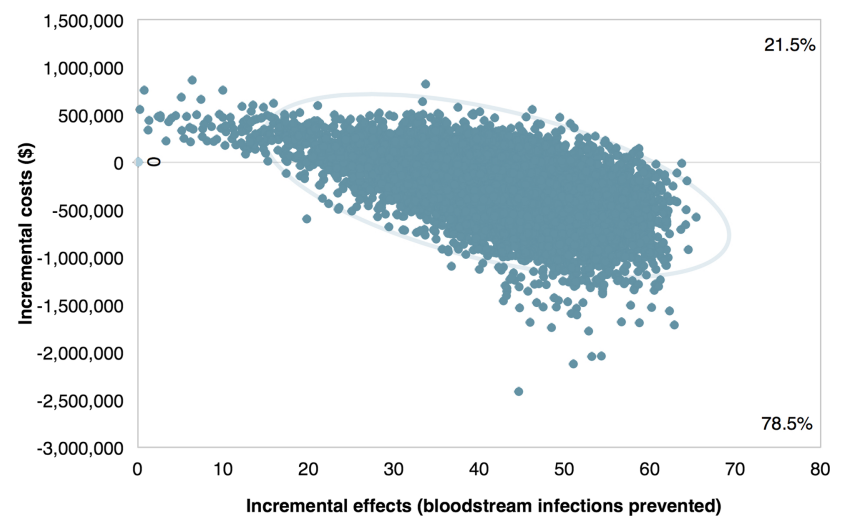

Figure 2 Cost-effectiveness plane for central line-associated bloodstream infections prevented with $95 \%$ confidence ellipses. Values on both axes have been multiplied by 1000 to yield incremental costs and effects expressed per 1000 patients to aid interpretation. Incremental refers to the difference in costs or effects between programme and non-programme intensive care units. Cost values in US dollars. Boxes in the plot region display the percentage of the distribution of incremental cost-effectiveness ratios falling above or below $\$ 0$. The $95 \%$ confidence ellipses overlaid on the figure are calculated assuming a bivariate normal distribution and display the uncertainty in the incremental costs-effectiveness ratios. 
outliers in the cost-effectiveness plane supports the robustness of the programme as a dominant strategy.

Online supplementary figures S2 and S3 display tornado diagrams of the results of the probabilistic sensitivity analysis. The opportunity cost of CLABSIs exerted the largest influence on the cost-effectiveness of the programme for preventing CLABSIs and deaths. As such, hospitals implementing the programme should know the opportunity costs they face due to CLABSIs. The opportunity cost is calculated as the potential incremental net revenue opportunity per prevented CLABSI. We provide an example of how to perform this calculation in the online appendix. Discounting CLABSIs or deaths at $3 \%$ in addition to costs, does not change the interpretation of our results. Similarly, discounting costs at $0 \%$ or $5 \%$ does not change the interpretation of our findings (see online appendix table $\mathrm{D}$ ).

\section{DISCUSSION}

This study represents the first formal cost-effectiveness analysis of a nationally implemented quality improvement programme (the Keystone ICU project) in the USA to decrease CLABSIs in critically ill patients. One of the few large scale quality improvement projects to demonstrate long-term sustainability, this programme has the potential to reduce CLABSIs and deaths at no additional cost to US hospitals.

\section{Comparison with other studies}

Previous studies highlight the importance and difficulty of developing a business case for quality improvement. A business case exists if healthcare organisations investing in an intervention reap a return on their investment. ${ }^{36}$ Many prevention initiatives have suffered from a lack of evidence supporting a positive return on investment for hospitals and payers. ${ }^{37}$ Incentive misalignment results in hospitals incurring costs to implement quality improvement programmes while payers accrue the subsequent cost savings. A financial analysis of a replication of the Keystone ICU project in one tertiary hospital in Hawaii demonstrated that reducing CLABSIs actually resulted in lower profit margins, thus creating a perverse incentive to provide a lower quality of care. ${ }^{38} \mathrm{~A}$ different study calculated that, for every CLABSI prevented, the programme costs $\$ 5404,{ }^{29}$ and suggested that it is cost saving when compared with the cost of an infection (which ranges from $\$ 6000$ to over $\$ 56000^{4} 6333940$ ). However, both studies used inaccurate cost analyses that focused on the financial rather than the economic costs of CLABSIs. These studies also did not account for uncertainty in the cost or effect estimates.

Our evaluation offers several improvements to these existing studies. First, whenever possible we used nationally representative data to determine provider salary and compensation costs, so as to increase the ability to generalise our findings. Second, we performed a probabilistic sensitivity analysis to quantify the uncertainty in our cost- effectiveness estimates. Third, we considered the opportunity costs of CLABSIs rather than financial costs. Our estimate of the cost of a CLABSI is based on the foregone hospital revenue that results whenever an infection occurs. From the perspective of the hospital, reducing the cost of CLABSIs is tantamount to reducing this foregone revenue by redeploying ICU beds for new admissions. Finally, we extended the evaluation to consider deaths prevented as an additional outcome because of its interest to clinicians and patients.

\section{Limitations of the study}

This evaluation has several limitations. First, the impact of patient heterogeneity, such as demographics and clinical characteristics on baseline risk, treatment effect or resource utilisation was not fully explored. ${ }^{41}$ This evaluation sought instead to represent an average ICU experience, in part because the data needed to explore subgroups are not available, but more importantly because the intervention applies to patients irrespective of these differences. It would be impractical-and possibly unethical-to only use this programme in subgroups of patients for whom greater benefit is expected. The programme is based on evidence-based practices for inserting central venous catheters-practices that should apply equally to all patients. Second, we did not evaluate costs outside the acute hospital setting, such as rehabilitation costs or productivity losses for delays returning to work incurred by patients suffering CLABSIs. However, doing so would further support the cost-effectiveness of the programme. We chose to examine costs from the perspective of the hospital because hospitals bear the greatest burden of nosocomial infection costs in the prospective payer system and demonstrating a business case is important for the dissemination of effective quality improvement programmes. Finally, this evaluation did not explore the use of antimicrobial catheters as an comparator because systematic reviews have come to differing conclusions about the extent of their effectiveness in preventing CLABSIs, and many of the trials have been small and of a low quality. ${ }^{43}$ In addition, the choice facing hospital decision makers is not necessarily a mutually exclusive choice between the Keystone ICU project or the use of antimicrobial catheters. The results of Keystone ICU project already reflect the use of various types of catheters because the programme itself did not specify catheter type. The parameter estimate for effectiveness of the programme used in this evaluation was derived from a cluster randomised controlled trial. In this setting it can be expected that the utilisation of antimicrobial catheters should be balanced between the intervention and control arms of the trial (though this was not explicitly measured), and the effectiveness estimate is attributable to the quality improvement programme itself. The Keystone ICU project quality improvement programme is also a complex social intervention. Comparing it directly to technology or a device 
understates its broader effects, which may include reductions in sepsis and ventilator-associated pneumonia or reduced staff turnover resulting from an improved safety culture. $^{21}$ Data for these effects are limited in comparison to the data available for CLABSIs, but recent evidence suggests that the Keystone ICU project significantly reduced rates of ventilator -associated pneumonia in Michigan ICUs. ${ }^{9}$ Inclusion of these additional beneficial effects for the same set of costs would further support the cost-effectiveness of the programme.

\section{Conclusions and implications of study findings}

These findings have important implications for healthcare. Broad implementation of the Keystone ICU project in the US healthcare system could substantially reduce the morbidity and mortality associated with CLABSIs and their economic costs to hospitals. Although the Centers for Disease Control and Prevention has demonstrated that significant reductions in CLABSIs are indeed being realised for intensive care patients in the USA, $^{44} 800$ medium and large hospitals continue to have high CLABSIs rates. ${ }^{45}$ Further dissemination of cost-effective quality improvement programmes is needed. Although our analysis adopted a hospital perspective, payers also stand to benefit from the programme and can support dissemination efforts. Hospitals and payers should partner to reform the incentive structure facing hospitals in order to better support patient safety and quality. Payer support, such as covering or funding some intervention costs and imposing financial penalties on hospitals when patients develop CLABSIs, could encourage uptake and dissemination of the programme. Future evaluations of this quality improvement programme in non-US settings can incorporate country-specific costs or extend the evaluation to consider additional outcomes, such as cases of ventilator-associated pneumonia prevented. As more data and evidence emerges regarding the long-term costs and outcomes for patients with CLABSI and other healthcare associated infections, ${ }^{46}$ future economic evaluations can incorporate this information to gauge the cost-effectiveness of prevention and quality improvement efforts.

To conclude, this multifaceted quality improvement programme, currently being implemented by thousands of hospitals in the USA, likely reduces unnecessary morbidity, mortality and economic costs associated with CLABSIs.

\author{
Author affiliations \\ ${ }^{1}$ Medical Scientist Training Program, Johns Hopkins School of Medicine, \\ Baltimore, Maryland, USA \\ ${ }^{2}$ Centre for Applied Health Research and Delivery (CAHRD), Liverpool School \\ of Tropical Medicine, University of Warwick, Coventry, UK \\ ${ }^{3}$ International Vaccine Access Center, Johns Hopkins Bloomberg School of \\ Public Health, Baltimore, Maryland, USA \\ ${ }^{4}$ Department of Health Policy and Management, Johns Hopkins Bloomberg \\ School of Public Health, Baltimore, Maryland, USA
}

${ }^{5}$ Armstrong Institute for Patient Safety and Quality, Johns Hopkins Medicine, Baltimore, Maryland, USA

Acknowledgements The authors thank Susan M. Turley for her assistance in editing the manuscript

Contributors $\mathrm{KRH}$ is the guarantor. All the authors were involved in preparing this manuscript. KRH was responsible for the overall study design, data analysis and interpretation of data, and wrote the initial draft of the manuscript. All other authors contributed to the study design, analysis and interpretation of data, and critical revision of the manuscript. LN, DOC, WJW and PJP also provided supervision.

Funding This research received no specific grant from any funding agency in the public, commercial or not-for-profit sectors.

Competing interests This study was conducted as part of KRH's doctoral training at the Johns Hopkins Bloomberg School of Public Health. KRH is supported by the Harry S. Truman Scholarship Foundation and by a training grant from the National Institutes of Health under award number T32 GM007309. PJP reports receiving grant or contract support from the Agency for Healthcare Research and Quality, the Gordon and Betty Moore Foundation (research related to patient safety and quality of care), the National Institutes of Health (acute lung injury research), and the American Medical Association Inc. (improved blood pressure control); honoraria from various healthcare organisations for speaking on patient safety and quality (the Leigh Bureau manages most of these engagements); and book royalties from the Penguin Group for his book Safe Patients, Smart Hospitals. PJP is a paid strategic advisor to the Gordon and Betty Moore Foundation; receives stock and fees to serve as a director for Cantel Medical; and is a founder of Patient Doctor Technologies, a start-up company that seeks to enhance the partnership between patients and clinicians with an application called Doctella.

Provenance and peer review Not commissioned; externally peer reviewed.

Data sharing statement No additional data are available.

Open Access This is an Open Access article distributed in accordance with the Creative Commons Attribution Non Commercial (CC BY-NC 4.0) license, which permits others to distribute, remix, adapt, build upon this work noncommercially, and license their derivative works on different terms, provided the original work is properly cited and the use is non-commercial. See: http:// creativecommons.org/licenses/by-nc/4.0/

\section{REFERENCES}

1. Mermel LA. Prevention of intravascular catheter-related infections. Ann Intern Med 2000;132:391-402.

2. Burke JP. Infection control-a problem for patient safety. $N$ Engl J Med 2003;348:651-6

3. Klevens RM, Edwards JR, Richards CL Jr, et al. Estimating health care-associated infections and deaths in U.S. hospitals, 2002. Public Health Rep 2007;122:160-6.

4. O'Grady NP, Alexander M, Dellinger EP, et al. Guidelines for the prevention of intravascular catheter-related infections. Infect Control Hosp Epidemiol 2002;23:759-69.

5. Digiovine B, Chenoweth $\mathrm{C}$, Watts $\mathrm{C}$, et al. The attributable mortality and costs of primary nosocomial bloodstream infections in the intensive care unit. Am J Respir Crit Care Med 1999;160:976-81.

6. Pittet D, Tarara D, Wenzel RP. Nosocomial bloodstream infection in critically ill patients. Excess length of stay, extra costs, and attributable mortality. JAMA 1994;271:1598-601.

7. Pronovost $\mathrm{P}$, Needham $\mathrm{D}$, Berenholtz $\mathrm{S}$, et al. An intervention to decrease catheter-related bloodstream infections in the ICU. $N$ Engl J Med 2006;355:2725-32.

8. Berenholtz SM, Pronovost PJ, Lipsett PA, et al. Eliminating catheter-related bloodstream infections in the intensive care unit. Crit Care Med 2004;32:2014-20.

9. Berenholtz SM, Pham JC, Thompson DA, et al. Collaborative cohort study of an intervention to reduce ventilator-associated pneumonia in the intensive care unit. Infect Control Hosp Epidemiol 2011;32:305-14.

10. Lin DM, Weeks K, Bauer L, et al. Eradicating central line-associated bloodstream infections statewide: the Hawaii experience. Am J Med Qual 2012;27:124-9.

11. Lipitz-Snyderman A, Steinwachs D, Needham DM, et al. Impact of a statewide intensive care unit quality improvement initiative on 
hospital mortality and length of stay: retrospective comparative analysis. BMJ 2011;342:d219.

12. Bion J, Richardson A, Hibbert $P$, et al. 'Matching Michigan': a 2-year stepped interventional programme to minimise central venous catheter-blood stream infections in intensive care units in England. BMJ Qual Saf 2013;22:110-23.

13. Marsteller JA, Sexton JB, Hsu YJ, et al. A multicenter, phased, cluster-randomized controlled trial to reduce central line-associated bloodstream infections in intensive care units. Crit Care Med 2012;40:2933-9.

14. Niessen LW, Grijseels EW, Rutten FF. The evidence-based approach in health policy and health care delivery. Soc Sci Med 2000;51:859-69.

15. Grimshaw JM, Thomas RE, MacLennan G, et al. Effectiveness and efficiency of guideline dissemination and implementation strategies. Health Technol Assess 2004;8:iii-iv, 1-72.

16. Hoomans T, Evers SM, Ament AJ, et al. The methodological quality of economic evaluations of guideline implementation into clinical practice: a systematic review of empiric studies. Value Health 2007;10:305-16.

17. Graves N, Harbarth S, Beyersmann J, et al. Estimating the cost of health care-associated infections: mind your p's and q's. Clin Infect Dis 2010:50:1017-21.

18. Veenstra DL, Saint S, Sullivan SD. Cost-effectiveness of antiseptic-impregnated central venous catheters for the prevention of catheter-related bloodstream infection. JAMA 1999;282: 554-60.

19. Halton KA, Cook DA, Whitby M, et al. Cost effectiveness of antimicrobial catheters in the intensive care unit: addressing uncertainty in the decision. Crit Care 2009;13:R35.

20. Needham DM, Scales DC, Laupacis A, et al. A systematic review of the Charlson comorbidity index using Canadian administrative databases: a perspective on risk adjustment in critical care research J Crit Care 2005;20:12-19.

21. Pronovost PJ, Berenholtz SM, Goeschel C, et al. Improving patient safety in intensive care units in Michigan. J Crit Care 2008;23:207-21.

22. Pronovost PJ, Berenholtz SM, Needham DM. Translating evidence into practice: a model for large scale knowledge translation. BMJ 2008;337:a1714.

23. Fletcher S. Catheter-related bloodstream infection. Contin Educ Anaesth Crit Care Pain 2005;5:49-51.

24. Brun-Buisson C. New technologies and infection control practices to prevent intravascular catheter-related infections. Am J Respir Crit Care Med 2001;164:1557-8.

25. Veenstra DL, Saint S, Saha S, et al. Efficacy of antiseptic-impregnated central venous catheters in preventing catheter-related bloodstream infection: a meta-analysis. JAMA 1999;281:261-7.

26. Martin MA, Pfaller MA, Wenzel RP. Coagulase-negative staphylococcal bacteremia. Mortality and hospital stay. Ann Intern Med 1989;110:9-16.

27. Smith RL, Meixler SM, Simberkoff MS. Excess mortality in critically ill patients with nosocomial bloodstream infections. Chest 1991:100:164-7.
28. Arnow PM, Quimosing EM, Beach $\mathrm{M}$. Consequences of intravascular catheter sepsis. Clin Infect Dis 1993;16:778-84.

29. Waters HR, Korn R Jr, Colantuoni E, et al The business case for quality: economic analysis of the Michigan Keystone Patient Safety Program in ICUs. Am J Med Qual 2011;26:333-9.

30. Graves N, Weinhold D, Tong E, et al. Effect of healthcare-acquired infection on length of hospital stay and cost. Infect Control Hosp Epidemiol 2007;28:280-92.

31. Graves N. Economics and preventing hospital-acquired infection Emerg Infect Dis 2004;10:561-6.

32. Society of Critical Care Medicine. Compensation of critical care professionals. Society of Critical Care Medicine, 2009.

33. Warren DK, Quadir WW, Hollenbeak CS, et al. Attributable cost of catheter-associated bloodstream infections among intensive care patients in a nonteaching hospital. Crit Care Med 2006;34:2084-9.

34. American Hospital Association. AHA Hospital Statistics. 2013 ed. American Hospital Association, 2012.

35. Pronovost PJ, Goeschel CA, Colantuoni E, et al. Sustaining reductions in catheter related bloodstream infections in Michigan intensive care units: observational study. BMJ 2010;340:c309.

36. Leatherman S, Berwick D, lles D, et al. The business case for quality: case studies and an analysis. Health Aff (Millwood) 2003;22:17-30

37. Galvin RS, Delbanco S, Milstein A, et al. Has the leapfrog group had an impact on the health care market? Health Aff (Millwood) 2005;24:228-33.

38. Hsu E, Lin D, Evans SJ, et al. Doing well by doing good: assessing the cost savings of an intervention to reduce central line-associated bloodstream infections in a Hawaii hospital. Am J Med Qual 2014;29:13-19.

39. Halton K, Graves N. Economic evaluation and catheter-related bloodstream infections. Emerg Infect Dis 2007;13:815-23.

40. Dimick JB, Pronovost PJ, Heitmiller RF, et al. Intensive care unit physician staffing is associated with decreased length of stay, hospital cost, and complications after esophageal resection. Crit Care Med 2001:29:753-8.

41. Grutters JP, Sculpher M, Briggs $A H$, et al. Acknowledging patient heterogeneity in economic evaluation : a systematic literature review. Pharmacoeconomics 2013;31:111-23.

42. Niel-Weise BS, Stijnen T, van den Broek PJ. Anti-infective-treated central venous catheters: a systematic review of randomized controlled trials. Intensive Care Med 2007;33:2058-68.

43. Hockenhull JC, Dwan K, Boland A, et al. The clinical effectiveness and cost-effectiveness of central venous catheters treated with anti-infective agents in preventing bloodstream infections: a systematic review and economic evaluation. Health Technol Assess 2008;12:iii-iv, xi-xii, 1-154.

44. Centers for Disease Control and Prevention. National and State Healthcare Association Infections Progress Report. Atlanta, 2014.

45. Wise ME, Scott RD II, Baggs JM, et al. National estimates of central line-associated bloodstream infections in critical care patients. Infect Control Hosp Epidemiol 2013;34:547-54.

46. Dick A, Liu H, Zwanziger J, et al. Long-term survival and healthcare utilization outcomes attributable to sepsis and pneumonia. BMC Health Serv Res 2012;12:432. 